\title{
DESEJO DE INFORMAÇÃO E PARTICIP AÇÃO NAS DECISÕES TERAPÊUTICAS EM CASO DE DOENÇAS GRAVES EM PACIENTES ATENDIDOS EM UM HOSPITAL UNIVERSITÁRIO
}

\author{
André Gulinelli, Rubens K. Aisawa, Sérgio N. Konno, Christian V. Morinaga, \\ Wagner l. Costardi, Rodrigo O. Antonio, Rodrigo B. Dumarco, Renata M. Moino, Márcio Katz, \\ Sandro Giavarotti, Allan P.Z. Skarbnik, Cyntia S. Forcione, toshio Chiba, Mílton A. Martins* \\ Trabalho realizado no Serviço de Clínica Geral do Hospital das Clínicas e no \\ Departamento de Clínica Médica da Faculdade de Medicina, Universidade de São Paulo, SP.
}

RESUMO - OвJetivo. Avaliar o desejo de pacientes serem informados sobre diagnóstico de doenças graves, de informação às suas famílias e de participação nas decisões terapêuticas.

Métodos. 363 pessoas atendidas no ambulatório ou internadas na enfermaria de um serviço universitário de Clínica Geral foram entrevistadas. $O$ questionário continha perguntas sobre desejo de ser informado e de que familiares também fossem informados em casos de diagnósticos de câncer e síndrome da imunodeficiência adquirida (Aids) e de ser informado e participar de decisões terapêuticas em caso de tumores abdominais.

Resultados. Homens $(96,1 \%)$ e mulheres $(92,6 \%)$ mostraram desejo de serem informados do diagnóstico de câncer e $87,7 \%$ dos homens e $84,2 \%$ das mulheres desejaram que sua família também fosse informada; $94,2 \%$ dos homens e $91 \%$ das mulheres afirmaram querer saber do diagnóstico de Aids. Enquanto $86 \%$ das mulheres e $76,6 \%$ dos homens mostraram desejo de serem informados das opções terapêuticas em caso de tumor abdominal, apenas 58,5\% das mulheres e $39,6 \%$ dos homens desejaram opinar sobre 0 tratamento. 0 desejo de participar das decisões terapêuticas foi menor nos homens, nas pessoas com mais de 60 anos e em quem estava internado $(p<0.05)$.

Conclusōes. A grande maioria da população que procura um hospital universitário deseja ser informada sobre suas condições de saúde, incluindo eventuais diagnósticos de doenças graves. Por outro lado, existem vínculos familiares intensos, sendo que os pacientes desejam, também, que suas famílias sejam informadas.

UnITERMOS: Câncer. Síndrome da imunodeficiência humana. Relação médico-paciente. Autonomia. Informação. Participação.

\section{INTRODUÇÃo}

A comunicação no cuidado médico abrange os conceitos de confidencialidade, relato da verdade, consentimento esclarecido e comportamento ético'. A informação ou relato da verdade é um dos conceitos médicos mais antigos, sendo que, em algumas culturas da antigüidade, a função do médico era, muitas vezes, mais informativa que curativa ${ }^{2}$. Já o consentimento esclarecido é um conceito bem mais recente, utiliza-se também da informação e está em sintonia com a importância da autonomia do paciente, paradigma estabelecido principalmente com o advento da Bioética moderna.

\footnotetext{
*Correspondência:

Departamento de Clínica Médica

Faculdade de Medicina da USP

Av. Dr. Arnaldo, 455 - Sala 1.216

São Paulo - SP - Cep: 01246-903

Tel. (11)3066.7317 - E-mail: mmartins@usp.br
}

A informação e o consentimento livre e esclarecido são considerados, em vários países ocidentais, como conceitos morais fundamentais. No Brasil estão previstos no Código de Ética Médica3 ${ }^{3}$ no artigo 59, que fala sobre a informação, e no artigo 56, que fala do consentimento esclarecido.

O relato da verdade tem mostrado ser um importante instrumento terapêutico. Suchman e Mattthews ${ }^{4}$ sugerem que a informação diminui o sentimento de isolamento do paciente e colabora para uma cooperação mútua na relação médico-paciente. Hall e colaboradores ${ }^{5}$ realizaram meta-análise de 4 I estudos e concluíram que a satisfação do paciente está relacionada à quantidade de informação recebida e à participação no tratamento.

Entretanto, estes conceitos não resultam em práticas universais de informação e participação de pacientes nas decisões que thes dizem respeito, inclusive em doenças graves como câncer e síndrome da imunodeficiência humana (Aids) 6,7. Muitas vezes não há informação sobre diagnóstico e prognóstico, com a justificativa de "proteção" ao paciente ou de não fazer o paciente sofrer com o diagnóstico. Por outro lado, existem diferenças culturais na forma de ver a doença e na expectativa em relação ao papel do médico ${ }^{7}$. Em um mesmo país, pode haver diferenças quando diferentes grupos étnicos são comparados. Estudo realizado por Blackhall et al. ${ }^{8}$, nos Estados Unidos, no estado da Califórnia, revelou que coreanos e mexicanos residentes nos EUA querem saber menos da verdade sobre o diagnóstico e prognóstico e participar menos da terapêutica do que os americanos com antepassados europeus ou africanos.

$\mathrm{Na}$ sociedade brasileira, freqüentemente, familiares tentam influenciar o médico sobre o tipo de informação que deve ser dado ao paciente 9 . Uma relação adequada com a família do paciente é, muitas vezes, decisiva para uma boa relação médico-paciente. Entretanto, 
poucos estudos realizados no Brasil tiveram como objetivo determinar com mais precisão o desejo das pessoas de serem informadas e participarem das decisões terapêuticas e, também, se essas informações deveriam ser dadas a membros da família. Uma vez que é possível haver diferenças significativas na visão sobre informação e participação em decisões médicas entre populações de diferentes países, consideramos importante que estudos em nosso meio sejam realizados.

O presente estudo teve como objetivos investigar o desejo que pacientes que utilizam um serviço universitário de atenção à saúde têm de serem informados em caso de diagnóstico de doenças graves e de participarem de decisões terapêuticas e o desejo de que seus familiares também sejam informados.

\section{Métodos}

O estudo foi realizado no Serviço de Clínica Geral do Hospital das Clínicas da Faculdade de Medicina da Universidade de São Paulo, tendo sido aprovado pela Comissão de Ética em Pesquisa da mesma instituição. Foram entrevistadas 363 pessoas, sendo 310 pacientes do ambulatório de Clínica Geral e 53 pacientes internados na enfermaria de Clínica Geral. Os entrevistados foram escolhidos de forma aleatória (sorteio), tanto no ambulatório, como na enfermaria. Os entrevistados no ambulatório eram pessoas com idade igual ou superior a 18 anos, escolhidas ao acaso entre os que estavam aguardando uma consulta médica no ambulatório de Clínica Geral. Os entrevistados na enfermaria de Clínica Geral, também escolhidos ao acaso, eram pacientes com idade igual ou superior a 18 anos, em condições clínicas estáveis e sem alterações cognitivas. Após ser explicado o objetivo da entrevista, era solicitado o consentimento do entrevistado e, então, a entrevista era realizada por um estudante de Medicina previamente treinado. A entrevista consistia de um questionário estruturado que continha, além da caracterização demográfica e socioeconômica do entrevistado, perguntas sobre o desejo de ser informado em caso de diagnóstico de câncer e de Aids, de que membro da família deveria ser informado caso houvesse o diagnóstico de câncer, e qual a opinião do entrevistado sobre quem deveria ser informado caso alguém de sua família (pai, mãe, filho e cônjuge) tivesse o diagnóstico de câncer. Havia, também, uma pergunta relacionada ao desejo de participar das decisões terapêuticas em caso de ser feito diagnóstico de um tumor abdominal. Neste caso, era perguntado se o paciente desejava ser informado dos riscos e benefícios dos diversos tratamentos possíveis em caso de estar doente (no caso, com um tumor abdominal) e, caso desejasse ser informado, se desejava opinar em relação à alternativa a ser escolhida pelo médico ou se preferia deixar esta decisão a cargo exclusivamente do médico. As perguntas do questionário estão no Anexo I.

Para a análise estatística foi empregado o programa SPSS 6.0 (SPSS Inc., EUA). Foi realizada, inicialmente, análise univariada, tendo como variáveis dependentes o desejo de ser informado de câncer ou de AIDS e o desejo de ser informado e de participar na decisão do tratamento de tumor abdominal. As variáveis independentes dos modelos foram o sexo, a idade, os anos de estudo, a religião, o estado civil e o fato de estar internado. Quando havia diferença estatisticamente significativa entre pelo menos uma das variáveis estudadas, era, a seguir, realizada uma análise multivariada (regressão logística). Um valor de $p$ inferior a 0,05 era considerado significativo.

\section{Resultados}

A Tabela I mostra as características das pessoas entrevistadas, quanto a sexo, faixa etária, anos de estudo, religião e estado civil. Foram consideradas casadas pessoas que tinham um parceiro estável residindo na mesma moradia.

A Figura I mostra a porcentagem de respostas às diversas alternativas da pergunta "Se você estivesse com câncer, quem gostaria que fosse avisado?". As três alternativas que implicam em desejo do entrevistado de ser informado pelo médico de um diagnóstico de câncer somadas correspondem a 96,1\% dos homens e $92,6 \%$ das mulheres. Por outro lado, a maioria dos entrevistados também mostrou desejo de que sua família fosse informada de um diagnóstico de câncer: as alternativas que implicavam na comunicação do diagnóstico, também ou exclusivamente à família, foram escolhidas por $87,7 \%$ dos homens e $84,2 \%$ das mulheres. $A$ análise univariada não revelou diferenças significativas do sexo, idade, anos de estudo, religião, estado civil ou o fato de estar internado no número de pessoas que mostraram desejo de serem informadas do diagnóstico de câncer.
As respostas à pergunta "se você estivesse com Aids quem você gostaria que fosse informado?", estão representadas na Figura 2. Como no caso da pergunta relativa ao câncer, a maioria dos entrevistados mostrou desejo de ser informado e de que sua família o fosse. A porcentagem de pessoas que desejaram ser informadas desse diagnóstico correspondeu a 94,2\% dos homens e $91,0 \%$ das mulheres, enquanto $78 \%$ dos homens e $74,5 \%$ das mulheres mostraram desejo de que a sua família também fosse informada. Não houve, também, influência significativa do sexo, faixa etária, anos de estudo, religião, estado civil e de estar internado nessas respostas.

A Tabela 2 mostra as porcentagens de respostas dadas às várias alternativas das perguntas relacionadas a quem deveria ser avisado em primeiro lugar em caso de membros de sua família com diagnóstico de câncer. As alternativas correspondentes a ninguém e a alguém indicado por esse familiar que estivesse doente não foram praticamente escolhidas. Não houve uma predominância nítida quando as outras alternativas foram avaliadas.

A Figura 3 mostra os resultados da pergunta que tinha por objetivo avaliar o desejo de ser informado de opções terapêuticas em caso de doença grave e o desejo de participar das decisões do médico relativas a essas alternativas terapêuticas ("tumor na barriga"). Nesse caso, mais uma vez, a maioria das pessoas mostrou desejo de ser informada: $86 \%$ das mulheres e $76,6 \%$ dos homens. Entretanto, apenas $58,5 \%$ das mulheres e $39,6 \%$ dos homens gostariam de opinar sobre o tratamento. A Tabela 3 mostra os resultados da análise multivariada realizada, em que a variável dependente foi o desejo de ser informado sobre as opções terapêuticas. Sexo masculino, idade superior a 60 anos e o fato do entrevistado estar internado foram associados significativamente a um maior desejo de não ser informado sobre as opções terapêuticas. A mesma análise foi realizada sobre o desejo de participar das decisões, incluindo na análise apenas aqueles que mostraram desejo de serem informados. A única variável que foi associada de forma significativa ao desejo de participar das decisões foi o sexo: uma porcentagem maior de homens mostrou desejo de deixar essas decisões a cargo apenas do médico. 
Anexol

ESTE QUESTIONÁRIO VISA SABER QUAL A SUA OPINIÃO SOBRE SITUAÇÕES QUE SERÃO APRESENTADAS. NÃO EXISTE RESPOSTA CERTA OU ERRADA. AS SITUAÇÖES APRESENTADAS SÃO FICTíCIAS.

Um paciente procurou um médico do Hospital da Clínicas e não sabia que doença tinha. Após a realização de vários exames, o médico pôde diagnosticar a doença.

I. Se o paciente fosse seu irmão e ele estivesse com câncer, quem deveria ser avisado primeiro do problema?

a) ninguém

b) ele mesmo

c) esposa ou companheira atual

d) você

e) outro membro da família - quem?

f) alguém indicado pelo seu irmão - quem?

2. Se o paciente fosse seu pai e ele estivesse com câncer, quem deveria ser avisado primeiro do problema?
a) ninguém
b) ele mesmo
c) esposa ou companheira atual
d) você
e) outro membro da família - quem?
f) alguém indicado pelo seu pai - quem?

3. Se o paciente fosse sua mãe e ela estivesse com câncer, quem deveria ser avisado primeiro do problema?
a) ninguém
b) ela mesma
c) esposo ou companheiro atual
d) você
e) outro membro da família - quem?
f) alguém indicado pela sua mãe - quem?

4. Se o paciente fosse seu filho adulto e ele estivesse com câncer, quem deveria ser avisado primeiro do problema?
a) ninguém
b) ele mesmo
c) esposa ou companheira atual
d) você
e) outro membro da família - quem?
f) alguém indicado pelo seu filho - quem?

5. Se o paciente fosse seu esposo ou esposa e ele estivesse com câncer, quem deveria ser avisado primeiro do problema?
a) ninguém
b) ele (ou ela) mesmo
c) a família dele (ou dela)
d) você
e) outro membro da família - quem?
f) alguém indicado por ele (ou ela) - quem?

6. Se você estivesse com Aids,
a) você gostaria de saber o que tem e que sua familia também soubesse
b) você gostaria de saber o que tem, mas que sua família não soubesse
c) você gostaria que sua família soubesse e, depois, alguém the contasse
d) você gostaria que sua família fosse avisada, mas não gostaria de saber da doença que você tem
e) você não gostaria que o médico contasse a ninguém qual doença você tem.

7. Se você estivesse com câncer,
a) você gostaria de saber o que tem e que sua família também soubesse
b) você gostaria de saber o que tem, mas que sua famillia não soubesse
c) você gostaria que sua familia soubesse e, depois, alguém the contasse
d) você gostaria que sua família fosse avisada, mas não gostaria de saber da doença que você tem
e) você não gostaria que o médico contasse a ninguém qual doença você tem.

8) Se você estivesse com um tumor na barriga, e o médico lhe falasse que tem três opções de tratamento igualmente eficazes,
a) você gostaria de saber das opções de tratamento e participar da decisão sobre o tratamento
b) você gostaria de saber das opções de tratamento e não gostaria de participar da decisão sobre o tratamento
c) você não gostaria de saber das opções de tratamento e deixaria o médico decidir sobre o tratamento 
GulinelLi A et Al.

\begin{tabular}{lccc}
\hline \multicolumn{4}{c}{ Tabela I - Características dos entrevistados (número de pessoas) } \\
\hline Sexo & masculino & 147 & $(40,5 \%)$ \\
& feminino & 216 & $(59,5 \%)$ \\
Idade (anos) & $<40$ & 129 & $(35,5 \%)$ \\
& 40 a 59 & 146 & $(40,2 \%)$ \\
Anos de estudo formal & $>60$ & 88 & $(24,2 \%)$ \\
& até 4 & 163 & $(44,9 \%)$ \\
Religiäo & mais do que 4 & 200 & $(50,1 \%)$ \\
& católicos & 253 & $(69,7 \%)$ \\
& evangélicos & 68 & $(18,7 \%)$ \\
Estado civil & outras & 15 & $(4,1 \%)$ \\
& sem religião & 27 & $(7,4 \%)$ \\
Local da entrevista & casados & 128 & $(35,3 \%)$ \\
& outros & 235 & $(64,7 \%)$ \\
& enfermaria & 53 & $(14,6 \%)$ \\
\end{tabular}

Figura I - Porcentagem dos entrevistados que escolheram cada uma das cinco alternativas de resposta à pergunta "Se você estivesse com câncer, quem gostaria que fosse avisado?

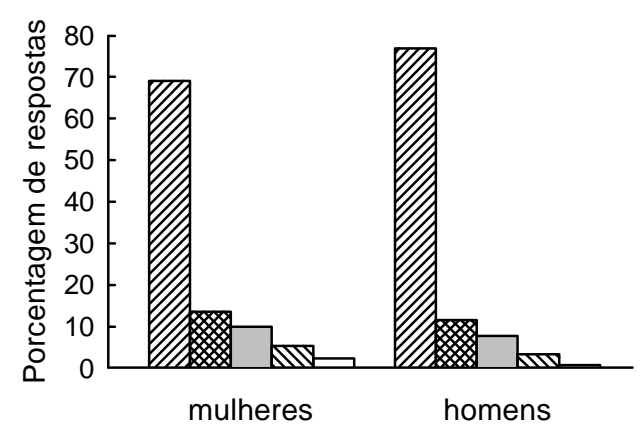

mostaria de saber e que sua família também soubesse

Gostaria de saber e que sua família não soubesse

$\square$ Gostaria que sua família soubesse e depois lhe contasse

Gostaria que sua família soubesse mas não gostaria de sabe

Gostaria que o médico não contasse a ninguém

\section{Discussão}

Observamos que a porcentagem de pacientes que desejam ser informados do diagnóstico de câncer e de Aids é muito alta, respectivamente $94 \%$ e $92,3 \%$. Além disso, a porcentagem dos que desejam que a família seja informada também é alta, respectivamente $85,6 \%$ e $75,9 \%$. Esses dados são semelhantes aos de países em que a maioria da população deseja ser informada sobre suas condições de saúde, mesmo em caso de doenças graves. Consideramos estudos como esses necessários e os resultados importantes para reforçar a idéia da informação das condições de saúde como um dever dos profissionais de saúde e um direito dos cidadãos. Em nosso questionário fizemos perguntas bem explícitas (câncer, Aids e "tumor na barriga) e não genéricas, para colocar os entrevistados perante situações bem concretas, para ficar bem claro o que está sendo perguntado.

Não encontramos trabalhos semelhantes ao nosso realizados no Brasil. Há trabalhos semelhantes realizados em outros países, com resultados bastante variados. Em estudo realizado na Grécia, 51,9\% dos entrevistados disseram desejar serem informados em caso de diagnóstico de câncer ${ }^{10}$. Quanto à informação, alguns estudos prévios mostraram que $87,3 \%$ dos pacientes são informados do diagnóstico de câncer nos EUA, 51,9\% na Itália e $29,5 \%$ no Japão6,11. A porcentagem encontrada em nosso estudo indubitavelmente mostra o desejo da amostra estudada em saber o diagnóstico. As altas porcentagens referentes à informação da família revelam um alto peso da família na relação médico-paciente.

É interessante ressaltar que a prática de informar o paciente em caso de diagnóstico de doença grave varia muito dependendo do país. Na Arábia Saudita'2, por exemplo, a sociedade é estruturada em unidades familiares, onde o homem mais velho toma as decisões; o consentimento para o tratamento do paciente é, geralmente, substituído pelo consentimento da família. No Irã' ${ }^{13}$, da mesma forma, é prática comum contar o diagnóstico de câncer somente a alguns membros da família. Na Uganda ${ }^{14}$, o diagnóstico de Aids é, com freqüência, omitido devido ao medo de reações psicológicas adversas nos pacientes. Na Grécia ${ }^{10}$, uma pesquisa realizada entre oncologistas e hematologistas mostrou que $73 \%$ nunca ou raramente informam o diagnóstico de câncer, e 95\% desses profissionais não informam o paciente em casos de morte iminente. No Japão"', a informação do diagnóstico de câncer aos pacientes é baixa $(29,5 \%)$ e a Suprema Corte Japonesa considera que o médico não é obrigado a informar a natureza de sua doença aos pacientes com câncer. Entretanto, há um esforço do Ministério de Saúde e Bem-estar Social do Japão em recomendar que se informe o diagnóstico e o prognóstico aos pacientes com câncer. Quanto à Aids", no Japão, das 168 instituições e hospitais que cuidam de paciente aidéticos, 66 (28\%) não informam o diagnóstico a seus pacientes. Na China ${ }^{15}$ não existe um código de ética médica e a perspectiva ética dos médicos é orientada por estudos culturais e religiosos e é prática comum informar somente à família no caso de câncer de mau prognóstico.

Quanto à informação terapêutica, notamos que a amostra estudada deseja ser informada da terapêutica $(82,2 \%)$, e que uma porcentagem mais baixa deseja 


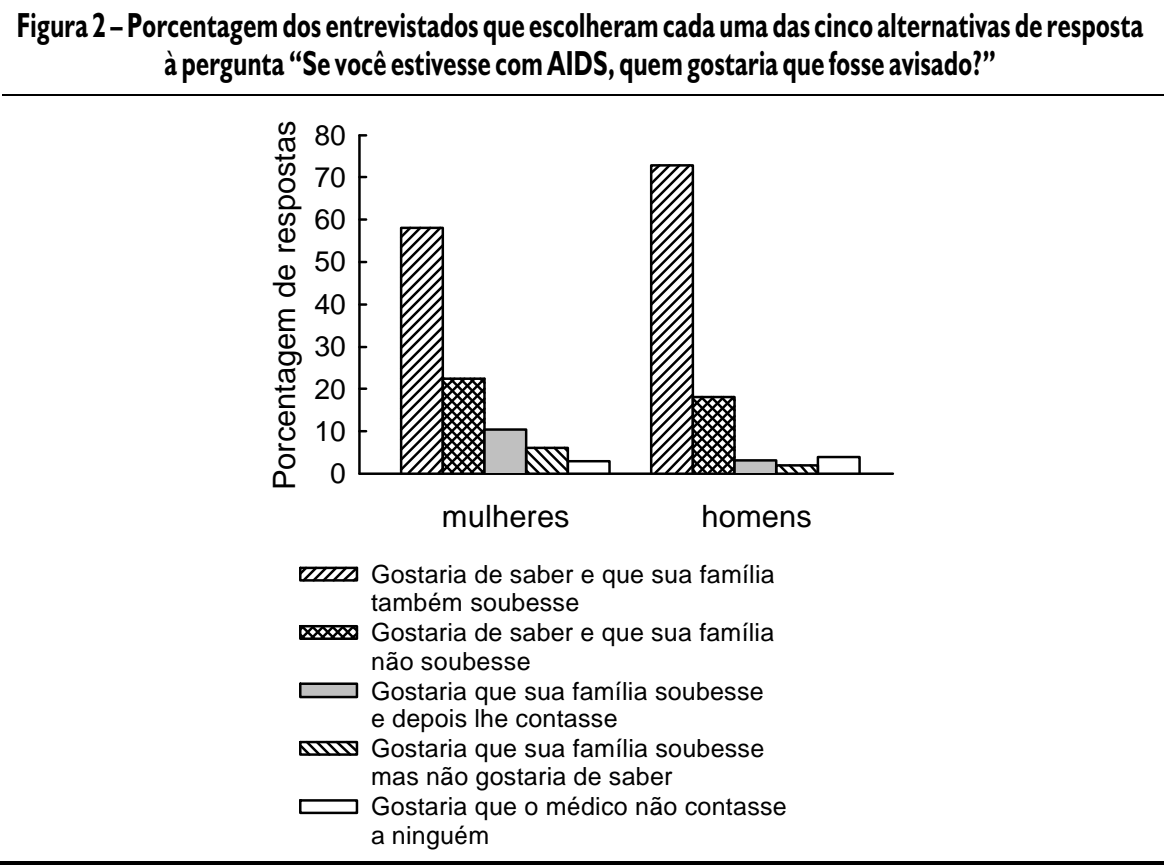

\begin{tabular}{|c|c|c|c|c|c|}
\hline \multicolumn{6}{|c|}{ Tabela 2 - Pessoa a ser avisada em primeiro lugar em caso de diagnóstico de câncer } \\
\hline \multirow[b]{2}{*}{$\begin{array}{l}\text { Membro da família a } \\
\text { ser avisado }\end{array}$} & \multicolumn{5}{|c|}{ MEMBRO DAFAMÍLIA COM CÂNCER } \\
\hline & $\begin{array}{l}\text { filho } \\
\text { (filha) }\end{array}$ & mãe & $\begin{array}{l}\text { esposo } \\
\text { (esposa) }\end{array}$ & pai & $\begin{array}{l}\text { irmão } \\
\text { (irmã) }\end{array}$ \\
\hline $\begin{array}{l}\text { Ninguém } \\
\text { Ele(a)mesmo(a) } \\
\text { Esposo(a) } \\
\text { O(a)entrevistado(a) } \\
\text { Outro membro da familila }\end{array}$ & $\begin{array}{l}0,0 \% \\
22,9 \% \\
18,0 \% \\
48,2 \% \\
10,9 \%\end{array}$ & $\begin{array}{l}0,0 \% \\
22,9 \% \\
22,4 \% \\
28,3 \% \\
26,4 \%\end{array}$ & $\begin{array}{c}0,5 \% \\
25,1 \% \\
67,5 \% \\
67,5 \% \\
6,9 \%\end{array}$ & $\begin{array}{l}0,0 \% \\
27,0 \% \\
25,4 \% \\
27,3 \% \\
20,3 \%\end{array}$ & $\begin{array}{l}0,0 \% \\
34,1 \% \\
22,4 \% \\
15,9 \% \\
27,4 \%\end{array}$ \\
\hline Total de respostas válidas & 356 & 363 & 362 & 362 & 359 \\
\hline
\end{tabular}

Figura 3 - Porcentagem dos entrevistados que escolheram cada uma das três alternativas de resposta quando 0 entrevistado era colocado na situação de alguém que tivesse um diagnóstico de "tumor na barriga" e que houvesse, para essa doença grave, mais de uma opção terapêutica

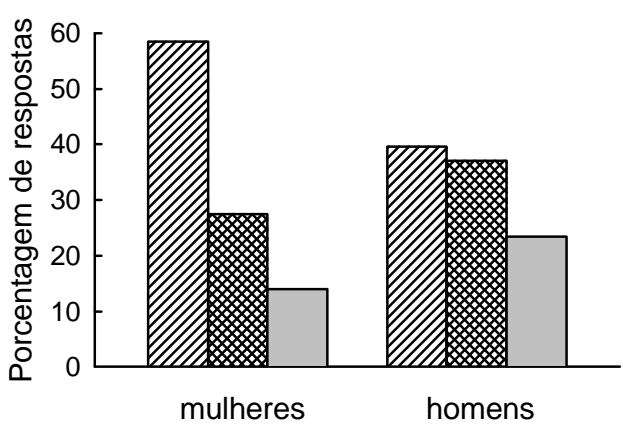

mostaria de saber das opções de tratamento e, também, de participar nas decisões

Gostaria de saber das opções de tratamento, mas não de participar nas decisões

Não gostaria de saber das opções de tratamento, deixando as decisões a cargo do médico participar da decisão terapêutica (50,8\%). Nos EUA ${ }^{8}$, uma pesquisa feita por questionários aplicados a pessoas saudáveis mostrou que, apesar da maior parte dos pacientes acreditar que deve saber da verdade sobre o prognóstico, apenas $41 \%$ dos entrevistados consideram que o paciente deve tomar as decisões quando o assunto é o uso de equipamento para prolongar a vida em doença incurável. Foi possível determinar que homens maiores de 60 anos têm um desejo menor de participar da terapêutica. Questionários aplicados nos EUA constataram que os pacientes jovens querem saber mais sobre diagnóstico e terapêutica e participar mais na terapêutica que os pacientes idosos ${ }^{8,16}$. Metaanálise de pesquisas utilizando gravações de entrevista ${ }^{17}$ concluiu que a equipe de saúde fornece mais informações a pacientes mulheres que a pacientes homens. Há alguns estudos que demonstram que as pacientes mulheres fazem mais perguntas em consultas que os pacientes homens ${ }^{17}$. Entretanto, Roter et al. ${ }^{17}$ observaram que a duração da consulta médica e o tempo dedicado a responder perguntas são influenciados tanto pelo sexo do médico quanto pelo do paciente, sendo mais longa a consulta de médicas consultando mulheres e a mais curta a de homens consultando mulheres.

No Brasil, também, nem sempre o diagnóstico é informado ao paciente. Nosso estudo demonstra que, em uma população que freqüenta um hospital universitário público, existe um desejo de informação. $O$ fato de não se informar o diagnóstico ao paciente pode ter alguns motivos: os médicos não alcançam uma relação médico-paciente que permita informar ao paciente ou não conseguem passar a informação de forma que possa ser entendida e, também, muitos pacientes não estão habituados a receber informações do médico e aceitam as decisões médicas sem questioná-las. Quanto ao primeiro aspecto, no México foi constatado que $80 \%$ dos estudantes de Medicina acreditam que o paciente deve ser informado do diagnóstico de câncer, entretanto, esse mesmo estudo 18 demonstrou que $72 \%$ destes estudantes evitavam utilizar a palavra "câncer" com o paciente. Cassileth et al..$^{16}$ em um estudo com 256 pacientes, verificaram que entre os pacientes que não eram 


\begin{tabular}{|c|c|c|c|c|}
\hline & & $\begin{array}{l}\text { Númerode } \\
\text { respostas } \\
\text { válidas }\end{array}$ & $\begin{array}{l}\text { Porcentagem } \\
\text { dos que não } \\
\text { querem saber }\end{array}$ & $\begin{array}{c}\text { Nível de } \\
\text { significância } \\
\text { (p) }\end{array}$ \\
\hline \multirow[t]{2}{*}{ Sexo } & feminino & 214 & $13,1 \%$ & \\
\hline & masculino & 146 & $24,7 \%$ & 0,023 \\
\hline \multirow[t]{3}{*}{ Idade (anos) } & $<40$ & 127 & $13,4 \%$ & \\
\hline & 40 a 59 & 144 & $15,3 \%$ & 0,733 \\
\hline & $>60$ & 89 & $28,1 \%$ & 0,032 \\
\hline \multirow[t]{2}{*}{ Anos de estudo } & até 4 & 162 & $17,9 \%$ & \\
\hline & mais do que 4 & 198 & $17,7 \%$ & 0,337 \\
\hline \multirow[t]{3}{*}{ Religião } & católicos & 250 & $20,0 \%$ & \\
\hline & evangélicos & 68 & $10,3 \%$ & 0,168 \\
\hline & outras & 42 & $16,7 \%$ & 0,868 \\
\hline \multirow[t]{2}{*}{ Estado civil } & casados & 125 & $15,2 \%$ & \\
\hline & outros & 235 & $19,1 \%$ & 0,675 \\
\hline Local & ambulatório & 307 & $13,7 \%$ & \\
\hline da entrevista & enfermaria & 53 & $41,5 \%$ & 0,001 \\
\hline
\end{tabular}

O nível de significância corresponde ao obtido na análise multivariada

\begin{tabular}{|c|c|c|c|c|}
\hline & & $\begin{array}{l}\text { Númerode } \\
\text { respostas } \\
\text { válidas }\end{array}$ & $\begin{array}{c}\text { Porcentagem } \\
\text { dos que não } \\
\text { querem participar }\end{array}$ & $\begin{array}{c}\text { Nível de } \\
\text { significância } \\
\text { (p) }\end{array}$ \\
\hline Sexo & $\begin{array}{l}\text { feminino } \\
\text { masculino }\end{array}$ & $\begin{array}{l}186 \\
110\end{array}$ & $\begin{array}{l}33,3 \% \\
48,2 \%\end{array}$ & 0,015 \\
\hline & 40 a 59 & 122 & $\begin{array}{l}35,6 \% 6 \\
42,6 \% \\
\end{array}$ & 0,421 \\
\hline Anos de estudo & até 4 & 133 & $45,1 \%$ & \\
\hline & mais do que 4 & 163 & $33,7 \%$ & 0,560 \\
\hline Religãáo & católicos & 200 & $41,0 \%$ & \\
\hline & evangélicos & 61 & $34,4 \%$ & 0,506 \\
\hline & outras & 35 & $34,3 \%$ & 0,817 \\
\hline & $\begin{array}{l}\text { casados } \\
\text { outros }\end{array}$ & $\begin{array}{l}106 \\
190\end{array}$ & $\begin{array}{l}38, / \% \\
38,9 \%\end{array}$ & 0,564 \\
\hline Local & ambulatório & 265 & $37,0 \%$ & \\
\hline da entrevista & enfermaria & 31 & $54,8 \%$ & 0,574 \\
\hline
\end{tabular}

O nível de significância corresponde ao obtido na análise multivariada

informados do diagnóstico, a maior parte gostaria de receber mais detalhes de sua doença. Truckett et al. ${ }^{19}$, em um estudo com 98 pacientes, verificaram que dois terços dos entrevistados possuíam perguntas que não foram feitas ao seu médico, pois achavam que havia tempo insuficiente e, portanto, ficaram relutantes em perguntar. Quanto ao segundo aspecto, nosso estudo permite afirmar que existe um desejo de informação e consentimento esclarecido por parte da população estudada, e esse aspecto deve ser enfatizado na formação de profissionais de saúde.
Em conclusão, na população estudada, existe um grande desejo de informação sobre diagnósticos médicos, em caso de doenças graves. Esse estudo reforça a idéia de que 0 paciente deve ser informado e convidado a participar das decisões terapêuticas que the dizem respeito. Por outro lado, para a maior parte dos pacientes entrevistados, a informação deve, também, ser dada à sua família. Informar o paciente de forma detalhada e compreensível e discutir com ele as opções terapêuticas devem ser habilidades e atitudes enfatizadas na formação médica.

\section{SUMMARY}

DESIRE OF INFORMATION AND PARTICIPATION IN THERAPEUTIC DECISIONS CONCERNING SEVERE DISEASES, IN PATIENTS OF A UNIVERSITY HOSPITAL

BACKGROUND. The purpose of our study was to evaluate the desire of the patients on being informed about diagnosis of severe diseases, the desire to have their families informed about this situation and to participate in therapeutic decisions.

METHODS. 363 patients (outpatients and inpatients) of a General Internal Medicine division of a University Hospital were interviewed. The questionnaire contained specific questions on their desire to be informed of the diagnosis in case of cancer or AIDS and on their desire to have their families informed as well. Specific questions on whether they wanted to be informed of and participate of the therapeutic discussion process in case of abdominal tumors were also included.

REsULtS. $96.1 \%$ of men and $92.6 \%$ of women showed the desire of being informed in case of cancer diagnosis and $87.7 \%$ of men and $84.2 \%$ of women wanted to have their families informed; $94.2 \%$ of men and $91 \%$ of women wanted to know the diagnosis of AIDS. While $86 \%$ of women and $76.6 \%$ of men wanted be informed in the case of a diagnosis of an abdominal tumor, only $58.5 \%$ of women and $39.6 \%$ of men wanted to give their opinion about in the case of different therapeutic alternatives. The desire to participate in therapeutic decisions was significantly lower $(p<0.05)$ in men, people older than 60 years and inpatients.

Conclusions. Our results showed that the great majority of the population that seeks for medical support in a Brazilian university hospital wishes to be informed on this health condition, even in case of serious illness. In addition, there are intense familiar bonds that make patients want to have their families also informed. [Rev Assoc Med Bras 2004; 50(I): 4I-7]

KEY wORDS: Cancer. Aids. Medical-patient relationship. Autonomy. Information. Participation. 


\section{REFERÊNCIAS}

I. Gonzalez G. Health Care in the United States, A perspective from the front line. Ann N Y Acad Sci 1997; 809:211-22.

2. Barona $X X$. Cancer patients and medical practice: some historical and cultural considerations. Ann N Y Acad Sci 1997; 809: 1 7-29.

3. Código de Ética Médica e textos sobre ética, direitos e deveres dos médicos e pacientes. São Paulo: Conselho Regional de Medicina do Estado de São Paulo; 2001.

4. Suchman AL, Matthews DA. What makes the patient-doctor relationship therapeutic? Exploring the connectional dimension of medical care. Ann Intern Med 1998; I 08: I 25-30.

5. Hall JA, Roter DL, Katz NR. Meta-analysis of correlates of provider behavior in medical encounters. Med Care 1988; 26:657-75.

6. Klocke JG, Klocker-Kaiser U, Schwaninger M. Truth in the relationship between cancer patient and physician. Ann N Y Acad Sci I 997; 809:56-65.

7. Pellegrino ED. Is truth telling to the patient a cultural artifact? JAMA 1992; 268: 1734-5.
8. Blackhall LJ, Murphy ST, Frank G, Michel V Azen S. Ethnicity and attitudes toward patient autonomy. JAMA 1995; 274:820-5

9. Faria SL, Souhami L. Communication with the cancer patient: information and truth in Brazil. Ann NY Acad Sci 1 997; 809: I 63-7| .

10. Fennelly JJ. Being honest with the patient and ourselves: Do we give our patients accurate insight into anticipated results of treatment? Ann N Y Acad Sci 1997; 809:383-92.

II. Mitsuya $\mathrm{H}$. Telling the truth to cancer patients and patients with HIV-I infection in Japan. Ann N Y Acad Sci 1997; 809:27989.

12. Youge D, Moreau P, Ezzat A, Gray A Communicating with cancer patients in Saudi Arabia. Ann N Y Acad Sci 1997 809:309-16.

13. Guavamzadeh A, Bahar B. Communication with the cancer patient in Iran. Ann N Y Acad Sci 1997; 809:26I-5.

14. Goodgame RW. AIDS in Uganda: Clinical and social features. N Eng J Med 1990; 323:383-9.
15. Li S, Chou JL. Communication with the cancer patient in China. Ann N Y Acad Sci 1997; 809:243-8.

16. Cassileth BR, Zupki RV, Sutton-Smith K, March $\checkmark$. Information and participation preferences among cancer patients. Ann Intern Med 1980; 92:832-6.

17. Roter D, Lipkin M, Korsgaard A. Sex differences in patients' and physicians' communication during primary care. Med Care 1991; 93:354-7.

18. Torrecillas L. Communication of the cancer diagnosis to mexican patients. Ann N Y Acad Sci 1997; 809: 1 88-96.

19. Truckett D, Williams A. Approaches to the measurement of explanation and information giving in medical consultations. Soc Sci Med 1984; | 8:57|-80

Artigo recebido: 23/09/02 Aceito para publicação: 05/08/03 